\title{
INTEGRAÇÃO ENSINO-SERVIÇO NA FORMAÇÃO DE PROFISSIONAIS PARA SISTEMAS PÚBLICOS DE SAÚDE
}

\author{
TEACHING-SERVICE INTEGRATION IN THE TRAINING OF \\ PROFESSIONALS FOR PUBLIC HEALTH SYSTEMS
}

\begin{abstract}
Lucas Balsanelli Souza ${ }^{1}$ (D) (0000-0001-8620-7894), Andréa Wander Bonamigo² (iD) (0000-0001-6435-704X)
${ }^{1}$ Universidade Federal de Ciências da Saúde de Porto Alegre, Programa de Pós-Graduação Ensino na Saúde, Porto Alegre, Rio Grande do Sul, Brasil. <ba.lucas@gmail.com>
\end{abstract}

${ }^{2}$ Universidade Federal de Ciências da Saúde de Porto Alegre, Programa de Pós-Graduação Ensino na Saúde, Departamento de Fonoaudiologia, Porto Alegre, Rio Grande do Sul, Brasil.

Resumo A integração ensino-serviço, entendida pelo trabalho articulado de estudantes, professores e trabalhadores em cenário de prática, tem por finalidades a excelência da formação profissional e a qualidade do trabalho em saúde. O objetivo deste estudo é analisar, por meio da percepção dos discentes, a integração ensinoserviço, que ocorre durante o período de graduação em Farmácia. Trata-se de um estudo quantitativo, conduzido em 2018, caracterizado como levantamento de corte transversal, no qual aplicou-se um questionário estruturado. Participaram do estudo 155 acadêmicos de cursos de Farmácia, matriculados em três universidades, duas públicas e uma privada, localizadas na região metropolitana de Porto Alegre, Rio Grande do Sul, Brasil. Os resultados encontrados sinalizaram as potencialidades dos estágios em cenários de saúde pública para a formação de profissionais farmacêuticos mais confiantes para o futuro trabalho no Sistema Único de Saúde. Os dados obtidos revelaram a variável idade dos discentes como fator relevante para o reconhecimento das competências necessárias para o trabalho. Tais achados podem contribuir para o processo de elaboração ou reformulação curricular dos cursos de Farmácia, com vistas à formação de um profissional crítico, reflexivo e conhecedor da realidade de saúde local.

Palavras-chave educação em saúde; educação em farmácia; serviços de integração docente-assistencial; Sistema Único de Saúde.
Abstract Teaching-service integration, understood through the combined work of students, teachers and workers in a practice setting, has as its goals excellency in professional training and quality of the work in health. The goal of the study is to analyze, through the perception of the students, the teaching-service integration, which takes place during the undergraduate Pharmacy studies. It is a quantitative study conducted in 2018, and characterized as a cross-sectional survey in which a structured questionnaire was applied. A total of 155 undergraduate Pharmacy students, enrolled in three different universities, a private university and two public ones, located in the metropolitan region of the city of Porto Alegre, in the state of Rio Grande do Sul, Brazil, participated in the study. The results found indicate the potentialities of the internships in public health settings for the training of Pharmacy professionals who are more confident regarding their future work at the Brazilian Unified Health System. The data obtained reveal the variable age of the students as a relevant factor for the acknowledgement of the competencies needed for the job. Such findings may contribute to the process of development or reformulation of the syllabi of the Pharmacy undergraduate courses, with the goal of training professionals who are critical, thoughtful and knowledgeable regarding the local health reality.

Keywords education in health; education in pharmacy; student-care integration services; Unified Health System. 


\section{Introdução}

O curso de graduação em Farmácia existe, no Brasil, desde 1832, quando foi formalmente criado, vinculado às escolas de medicina do Rio de Janeiro e da Bahia. Desde então, observa-se contínuo aumento no número de cursos habilitados e de vagas ofertadas. Em 2004 havia no país, 237 cursos habilitados (Spada et al., 2006). Entre 2005 e 2018, houve uma ampliação de quase $190 \%$, atingindo, ao final deste período, o total de 675 cursos de Farmácia habilitados no Brasil. A esta ampliação correspondeu o aumento de vagas ofertadas, as quais passaram de 27.769, no ano de 2004, para 98.165, em 2018 , equivalente a um crescimento de mais de $250 \%$, que se mostrou significativamente maior no segmento privado de ensino (Brasil, 2018a).

Além da expansão do curso, também ocorreram, neste período, alterações nas estruturas curriculares, aproximando-o às necessidades de saúde da população brasileira. Na década de 1930, iniciaram-se mudanças curriculares com o objetivo da formação de um profissional farmacêutico que estivesse habilitado, tanto ao exercício das atividades de Farmácia quanto ao desempenho em atividades na indústria e em laboratórios clínicos. Em 1962, materializada a proposição do primeiro currículo mínimo, foram implementadas disciplinas voltadas para os laboratórios de análises clínicas. Em 1969, a estrutura curricular foi dividida em ciclos profissionais, correspondentes às modalidades: farmacêutico, farmacêutico industrial e farmacêutico bioquímico (Spada et al., 2006).

Com o surgimento do Sistema Único de Saúde (SUS), no final da década de 1980, e as consequentes e profundas mudanças que ocorreram na área da saúde, estudantes, professores e trabalhadores retomaram as discussões sobre a identidade e a formação do profissional farmacêutico. Neste mesmo período, foi elaborada e promulgada a Lei de Diretrizes Básicas da Educação (LDB), que propunha a substituição dos currículos mínimos pelas Diretrizes Curriculares dos Cursos. Com a vigência desta lei, as Instituições de Ensino Superior (IESs) adquiriram mais autonomia, podendo elaborar e fixar seus currículos desde que observadas as diretrizes gerais pertinentes aos cursos (Brasil, 1996). Ao recuperar as discussões levadas a efeito no período anterior, a comunidade farmacêutica propôs diretrizes gerais para a educação farmacêutica no Brasil (Spada et al., 2006).

Em 2002, a resolução CNE/CES n. 2 de 2002 elaborou as primeiras Diretrizes Curriculares Nacionais (DCNs) para os cursos de graduação em Farmácia no país, propondo a transição da formação especializada para a formação generalista, humanista, crítica e reflexiva. Vigentes pelo período de 15 anos, as DCNs reforçaram a necessidade da interação dos discentes com as necessidades sociais da saúde e de uma formação farmacêutica direcionada para o SUS (Brasil, 2002). Após esse período de vigência, novas DCNs foram for- 
muladas, estabelecendo novos marcos para a formação superior em Farmácia. Estas preconizam a formação de profissionais que respondam aos desafios das sociedades contemporâneas e que incorporem uma visão mais aprofundada dos problemas sociais brasileiros. (Brasil, 2017).

As DCNs de 2017 para os cursos de graduação em Farmácia, expressas na resolução n. 6, de 19 de outubro de 2017, mantiveram a formação humanista, crítica, reflexiva e generalista e apresentaram importantes normativas para as relações entre ensino-serviço-comunidade. Suas normas explicitam a participação das IESs no processo de integração ensino-serviço-comunidade; valorizam os estágios curriculares em semestres iniciais dos cursos, devendo os estágios obrigatórios contemplar cenários de prática do SUS, nos diversos níveis de complexidade; e atribuem às IESs a responsabilidade de fomentar a educação permanente dos profissionais da rede de saúde, com vistas à melhoria do serviço e do processo de ensino-aprendizagem nos cenários de práticas (Brasil, 2017).

Contudo, pouco se tem problematizado a integração ensino-serviço e suas contribuições no processo de formação superior em Farmácia (Souza e Bonamigo, 2018). Permanecem sem respostas diversas questões, por exemplo, como ocorre a integração ensino-serviço durante o período de graduação em Farmácia? Quais as contribuições que as atividades de integração entre ensino e serviços públicos de saúde oferecem ao processo de formação acadêmica? Este estudo visou responder estas questões.

\section{Desenho metodológico}

Trata-se de um estudo quantitativo ${ }^{1}$, caracterizado como levantamento de corte transversal, que possibilitou apresentar uma descrição quantitativa e numérica de tendências, atitudes, percepções e opiniões da população estudada (Creswell, 2010).

A coleta e a elaboração dos dados ocorreram nos meses de agosto, setembro e novembro de 2018, por meio do preenchimento de um questionário estruturado, constituído por 38 questões elaboradas pelos autores do presente estudo. Para as questões de percepção foram utilizadas afirmativas apresentando graus de concordância (escala do tipo Likert, 1 - menor grau de concordância, 5 - maior grau de concordância). O questionário foi disponibilizado em sala de aula.

As informações referentes às análises documentais foram obtidas por meio de documentos disponibilizados pelas IESs, nos respectivos websites institucionais. Todas as informações foram posteriormente confirmadas, por e-mail, com as coordenações dos cursos participantes desta investigação.

Os participantes da pesquisa foram acadêmicos de Farmácia de três universidades (IES1, IES2 e IES3) localizadas na região metropolitana de Porto 
Alegre (RMPA). Todos, maiores de 18 anos de idade, estavam cursando o terceiro, o quarto ou o último ano da graduação e encontravam-se presentes, em sala de aula, no momento da aplicação do questionário. Responderam o questionário somente os acadêmicos que aceitaram participar da pesquisa mediante assinatura do termo de consentimento livre e esclarecido.

Os resultados foram apresentados por meio de análise descritiva, em distribuição das frequências e associações, avaliadas pelo teste qui-quadrado de Pearson e verificadas com auxílio dos resíduos padronizados ajustados. Para as questões com grau de concordância, os escores foram descritos em média e desvio padrão ( $\mathrm{dp}$ ) e suas diferenças avaliadas usando a Anova com teste post-hoc de Tukey.

Para avaliar a força de associação das variáveis na alteração do conjunto das questões relativas a conhecimentos e habilidades para a atuação no SUS, os participantes da pesquisa foram classificados em dois grupos com base no somatório das respostas do módulo. Definiu-se como integrantes do grupo com maiores habilidades e conhecimentos para o trabalho no SUS os respondentes com pontuação acima de 24 pontos. Foram aplicados os modelos de regressão de Poisson para as análises bivariadas e multivariadas e apresentaram-se as medidas de razão de prevalência (RP) com intervalo de confiança de $95 \%$ (IC95\%). As análises foram realizadas no software SPSS versão 23 e a significância estatística adotada foi de 5\%.

Esta pesquisa, oriunda da dissertação de mestrado profissional no Ensino na Saúde, foi aprovada pelo Comitê de Ética em Pesquisa com Seres Humanos (CEP) da Universidade Federal de Ciências da Saúde de Porto Alegre (UFCSPA), mediante o parecer n. 2.733.765.

\section{Resultados}

Três IESs participaram do estudo, duas públicas e uma privada. Duas delas desenvolvem as atividades acadêmicas no turno noturno (IES1, IES2) e uma, em turno integral (IES3). O curso de turno integral corresponde ao de menor carga horária total $(4.475 \mathrm{~h})$. Os cursos noturnos têm carga horária total de $4.831 \mathrm{~h}$ (IES2) e $5.140 \mathrm{~h}$ (IES1).

Participaram do estudo, voluntariamente, 155 discentes de graduação em Farmácia: 41 (26,4\%) da IES1; 39 (25,2\%) da IES2; 75 (48,4\%) da IES3. No quesito ensino privado ou público, 116 respondentes $(74,8 \%)$ pertenciam às instituições públicas e $39(25,2 \%)$, à instituição privada. Na Tabela 1 , caracterizam-se os participantes deste estudo. 
Tabela 1

Caracterização dos discentes do curso de Farmácia, por instituição de ensino superior, considerando faixa etária, gênero, vínculo empregatício e período do curso. Região metropolitana de Porto Alegre, RS, Brasil, 2018.

\begin{tabular}{|c|c|c|c|c|c|c|c|c|}
\hline \multirow[t]{2}{*}{ Universidades } & \multicolumn{2}{|c|}{ IES1 } & \multicolumn{2}{|c|}{ IES2 } & \multicolumn{2}{|c|}{ IES3 } & \multicolumn{2}{|c|}{ Total } \\
\hline & $\mathbf{n}$ & $\%$ & $\mathbf{n}$ & $\%$ & $\mathbf{n}$ & $\%$ & $\mathbf{n}$ & $\%$ \\
\hline \multicolumn{9}{|c|}{ Faixa etária (anos) } \\
\hline $18-25$ & 25 & $(61,0)$ & 19 & $(50,0)$ & 49 & $(67,1)$ & 93 & $(61,2)$ \\
\hline $26-35$ & 13 & $(31,7)$ & 15 & $(39,5)$ & 23 & $(31,5)$ & 51 & $(33,6)$ \\
\hline $36-45$ & 3 & $(7,3)$ & 4 & $(10,5)$ & 1 & $(1,4)$ & 8 & $(5,3)$ \\
\hline
\end{tabular}

Gênero

0,188

$\begin{array}{lllllllll}\text { Masculino } & 9 & (22,0) & 6 & (15,4) & 10 & (13,3) & 25 & (16,1) \\ \text { Feminino } & 32 & (78,0) & 33 & (84,6) & 65 & (86,7) & 130 & (83,9)\end{array}$

Vínculo Empregatício

0,478

\begin{tabular}{|c|c|c|c|c|c|c|c|c|c|}
\hline Emprego & 8 & $(21,6)$ & 21 & $(67,7)$ & 7 & $(13,2)$ & 36 & $(29,8)$ & \\
\hline Somente estudante & 29 & $(78,4)$ & 10 & $(32,3)$ & 46 & $(86,8)$ & 85 & $(70,2)$ & \\
\hline Período do curso & & & & & & & & & 0,008 \\
\hline $3^{\circ}-4^{\circ}$ ano & 29 & $(70,7)$ & 14 & $(35,9)$ & 39 & $(52,0)$ & 82 & $(52,9)$ & \\
\hline Último ano & 12 & $(29,3)$ & 25 & $(64,1)$ & 36 & $(48,0)$ & 73 & $(47,1)$ & \\
\hline
\end{tabular}

Fonte: Os autores.

IES: instituição de ensino superior 
Na comparação da amostra entre as IESs, tiveram diferenças estatísticas significantes as variáveis: vínculo empregatício ( $p<0,001)$, IES2 associada a discentes com vínculo empregatício $(67,7 \%)$ e a IES3 àqueles sem vínculo empregatício $(86,8 \%)$; período do curso (p-valor 0,008), IES1 associada a discentes do $3^{\circ}$ ao $4^{\circ}$ ano $(70,7 \%)$ e IES2 aos do último ano $(64,1 \%)$. Nas demais categorias não houve diferenças (Tabela 1). Embora 155 participantes tenham respondido o questionário, ao contabilizar as variáveis separadamente, observou-se variação no número de eventos devido à ausência de respostas em algumas das questões analisadas.

\section{Formação para o Sistema Único de Saúde}

A análise das matrizes curriculares mostrou que existem diferenças entre os cursos de Farmácia quanto à nomenclatura, à oferta, à carga horária e ao período em que são ofertadas as disciplinas relacionadas à formação para o SUS. Quanto à carga horária, a IES1 oferecia 120 horas $(2,33 \%$ da carga horária total do curso) de disciplinas teórico/práticas relacionadas à formação para o SUS; a IES2 oferecia 120 horas (2,48\% da carga horária total do curso); a IES3 oferecia 135 horas (3,01\% da carga horária total do curso).

Ao serem questionados sobre as disciplinas que abordam temáticas referentes ao SUS, como histórico, organização e modelo de atenção à saúde, $100 \%$ (155) dos discentes responderam que, durante a graduação, cursaram tais disciplinas. Algo semelhante ocorreu com a participação dos discentes em disciplinas que tratam da assistência farmacêutica (AF) no SUS, sua organização e o papel do farmacêutico. Um total de 98,1\% (152) dos discentes respondeu que o curso de Farmácia aborda estas temáticas durante a formação acadêmica. Para ambas as questões não houve diferenças significativas entre as IESs.

Os discentes foram questionados se durante a graduação cursaram disciplinas obrigatórias ou eletivas juntamente com outros cursos da saúde. Houve, entre as três IESs, diferenças significativas nas respostas ( $<<0,001)$. Maior integração acadêmica entre diferentes profissões, proporcionada por disciplinas com alunos de diferentes cursos da saúde, ocorreu nas IES1 e IES2, nas quais, respectivamente, um total de 92,7\% (38) e 94,9\% (37) dos discentes cursaram disciplinas no formato descrito. Na IES 3, 61,3\% (46) dos discentes relataram tal integração.

Observa-se, por estes resultados, uma tentativa de formação farmacêutica integrada às demais profissões da saúde, possibilitando aos discentes de Farmácia contato com outros profissionais da saúde ainda em ambiente acadêmico. Proporcionar o convívio simultâneo entre as diferentes profissões da saúde abre espaço para a introdução de modelos de formação condizentes com os princípios e as diretrizes do SUS. A citar, o modelo de formação multiprofissional, que ocorre de modo paralelo, entre discentes de duas ou mais 
profissões, sem haver interação; e o modelo de formação interprofissional, com interação e aprendizagem compartilhada (Peduzzi et al., 2013).

Educação interprofissional (EIP) é uma modalidade de formação em saúde que promove o trabalho em equipe, integrado e colaborativo, entre profissionais de diferentes áreas, com a finalidade de melhorar as respostas dos serviços e a qualidade da atenção à saúde (Peduzzi et al., 2013). Esta modalidade vem sendo empregada como estratégia de ensino na saúde, com vistas à formação de profissionais críticos, reflexivos, capazes de trabalhar em equipe e de responder às necessidades sociais de saúde (Faria et al., 2018). Para a consecução de tais propósitos, o Ministério da Saúde, por meio do Edital n. 10, de 23 de julho 2018, abriu inscrições para o PET-Saúde Interprofissionalidade, com o propósito de aprimorar as práticas colaborativas em saúde (Brasil, 2018b).

Acerca dos estágios curriculares, as três IESs ofertam, em suas grades curriculares, estágios em saúde pública. Entretanto, eles não são obrigatórios em todos os cursos analisados. Há acadêmicos de Farmácia que concluem sua formação sem antes ter vivenciado a realidade local de saúde, mesmo que parcialmente. Dos discentes que estagiaram nos serviços do SUS, pouco mais de $25 \%$ realizaram atividades tanto na atenção básica $(\mathrm{AB})$ quanto na média e alta complexidade (MAC) do SUS. Atualmente, há o predomínio da AB $(65,7 \%)$ como cenário de prática dos estágios em saúde pública.

Para alcançar a formação proposta pelas DCNs 2017 - humanista, crítica, reflexiva e generalista - e capacitar o farmacêutico para o trabalho nos diferentes níveis de complexidade do sistema de saúde (Brasil, 2017), o discente deve vivenciar o serviço em sua integralidade e em seus diversos níveis de complexidade, visto que disciplinas teórico-práticas, apesar da reconhecida importância no processo ensino-aprendizagem, não são capazes de sozinhas prepararem tal profissional (Ceccim, Bravin e Santos, 2009). Para alcançar os objetivos referidos, são necessárias a reavaliação e a reformulação curriculares, assim como são requeridos novos currículos que, idealmente, oportunizem e valorizem o maior contato dos discentes com os cenários de prática do SUS.

\section{Integração ensino e serviços públicos de saúde}

O estudo desenvolvido abordou a integração entre ensino e serviços públicos de saúde (Tabela 2) e diferenças significativas entre as IESs foram encontradas nas três modalidades: participação do discente em atividades de ensino com visitas aos serviços de saúde - a IES3 foi associada à maior participação dos discentes nestas atividades $(70,7 \%)$; realização de estágios obrigatórios - a IES2 foi associada à maior participação dos discentes nesta modalidade de estágio (61,5\%); realização de estágios não obrigatórios - a IES1 foi associada à maior participação dos discentes nesta modalidade $(58,5 \%)$. 
Tabela 2

Caracterização das vivências dos discentes do curso de Farmácia em serviços públicos de saúde, considerando as instituições de ensino superior e as modalidades de contato com os serviços. Região metropolitana de Porto Alegre, RS, Brasil, 2018.

\begin{tabular}{|c|c|c|c|c|c|}
\hline \multirow[t]{2}{*}{ Universidades } & IES1 & IES2 & IES3 & Total & p-valor \\
\hline & n (\%) & n (\%) & n (\%) & n (\%) & \\
\hline
\end{tabular}

0,001

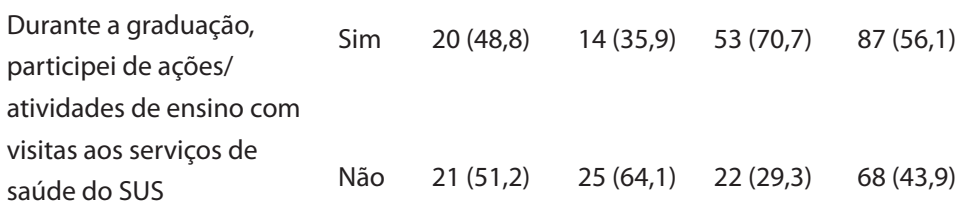

0,988

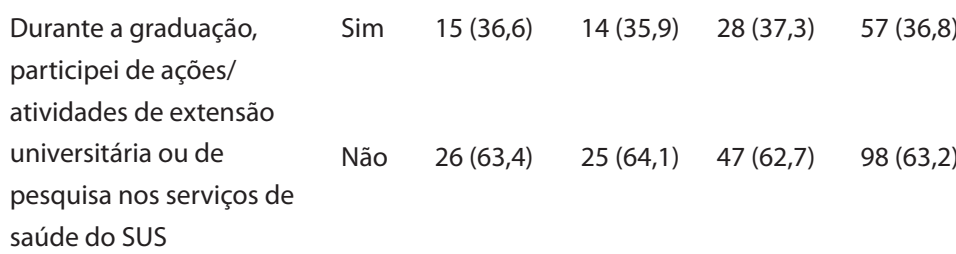

$\begin{array}{llllll}\begin{array}{l}\text { Durante a graduação, } \\ \begin{array}{l}\text { realizei estágio(s) } \\ \text { obrigatório(s) nos }\end{array}\end{array} & \text { Sim } & 6(14,6) & 24(61,5) & 30(40,0) & 60(38,7) \\ \begin{array}{l}\text { serviços de saúde do SUS } \\ \text { Não }\end{array} & 35(85,4) & 15(38,5) & 45(60,0) & 95(61,3)\end{array}$

\begin{tabular}{|c|c|c|c|c|c|}
\hline $\begin{array}{l}\text { Durante a graduação, } \\
\text { realizei estágio(s) não }\end{array}$ & Sim & $24(58,5)$ & $24(32,0)$ & $14(35,9)$ & $62(40,0)$ \\
\hline $\begin{array}{l}\text { obrigatório nos serviços } \\
\text { de saúde do SUS }\end{array}$ & Não & $17(41,5)$ & $51(68,0)$ & $25(64,1)$ & $93(60,0)$ \\
\hline
\end{tabular}

Fonte: Os autores.

SUS: Sistema Único de Saúde; IES: instituição de ensino superior

Na análise global das vivências nos serviços públicos de saúde, verificou-se que $61,9 \%$ (96) dos discentes realizaram, durante seu período de graduação, no mínimo uma das modalidades de estágios no SUS. Ao considerar todas as modalidades de vivência nos serviços, citadas anteriormente, notou-se que 79,4\% (123) dos discentes vivenciaram os serviços públicos por meio de uma ou mais das modalidades. Em ambas as análises, não houve diferenças significativas entre as IESs. Observa-se, por estes resultados, que ainda há espaço 
para ampliar a inserção dos discentes em cenários de prática no SUS, por meio da oferta de estágios ou de outras modalidades de vivência nos serviços.

Percebeu-se que algumas IESs mantêm o estágio curricular apenas nos últimos semestres do curso e que nem todas inserem, em suas grades curriculares, estágios obrigatórios em cenários de prática do SUS. Em consequência, o discente pode egressar da instituição de ensino sem ter tido seu primeiro contato com a saúde pública. As novas DCNs, de 2017, para os cursos de graduação em Farmácia vieram modificar este cenário. Elas orientam a formação de profissionais capacitados para o trabalho nos diferentes níveis de complexidade do sistema de saúde e determinam o início dos estágios obrigatórios, no máximo, no terceiro semestre do curso (Brasil, 2017).

Segundo Machado e Neto (2018), faz-se necessária uma educação na saúde voltada para a qualidade do cuidado, pautada nos determinantes sociais da saúde, nas necessidades da população, no perfil epidemiológico local e que desenvolva um currículo que preveja a inserção do estudante de graduação em cenários de práticas, desde os primeiros semestres do curso. Para Faria et al. (2018), uma maneira de fortalecer a formação profissional em saúde é reafirmar o compromisso social das IESs com a comunidade. Este compromisso contribui para que os projetos de extensão e/ou pesquisa sejam construídos conjuntamente, envolvendo profissionais, comunidade e estudantes em propostas coerentes com a realidade local e que atendam aos princípios do sistema de saúde.

As relações dos discentes com supervisores e preceptores de estágios foram analisadas sob a perspectiva da integração ensino-serviço. Com relação à supervisão de estágio, observou-se maior presença do(a) preceptor(a) de estágio no acompanhamento do estagiário $(88,8 \%)$, quando comparada com a presença do(a) professor(a) da IES (40,2\%). O acompanhamento do estágio por ambos os profissionais, como preconizado para estágios curriculares, foi considerado insuficiente. Somente 38,8\% dos discentes de Farmácia relataram a presença de ambos os profissionais como supervisores ativos em suas atividades práticas de estágio.

Apesar da variável supervisão de estágio não ter alterado significativamente a autopercepção dos discentes quanto ao preparo para a atuação nos serviços públicos de saúde, a ausência do supervisor de estágio tem potencial para limitar as capacidades formativas das atividades desta etapa da formação. A ação pedagógica do docente tem relação direta com a formação de perfis profissionais para atender às necessidades do SUS. Para Damiance et al. (2016), o docente supervisor de estágio é um dos responsáveis por proporcionar aos estagiários experiências de aprendizado significativas e transformadoras das práticas profissionais.

Os resultados do estudo ora exposto mostraram a participação ativa do preceptor de estágio nas vivências dos discentes em saúde pública. O far- 
macêutico preceptor de estágio foi reconhecido pelos discentes pelo efetivo acompanhamento das atividades em locais de serviço. Contudo, Cavalheiro e Guimarães (2011), ao citarem as dificuldades para a efetivação da integração ensino-serviço-comunidade, mencionam a resistência dos profissionais à inserção dos estudantes nos serviços, tanto por não julgarem condizente com a agenda de trabalho quanto pelo receio de identificação de suas fragilidades. Portanto, além de se contar com a participação ativa do farmacêutico preceptor de estágio, é necessário que se criem condições dentro dos processos de trabalho para que ele possa exercer seu papel de educador, contribuindo com a integração ensino-serviço-comunidade.

\section{Contribuições dos estágios em saúde pública para a formação em Farmácia}

Os discentes de Farmácia mostraram lograr benefícios da dinâmica cotidiana em serviço, sobretudo das atividades de formação que ocorrem nos cenários de prática do SUS. Aqueles que realizaram estágios no SUS demonstraram alto grau de concordância com as afirmações referentes às contribuições dos estágios para o processo de formação. Segundo eles, os estágios contribuem indiscutivelmente para a formação acadêmica $(4,69 \mathrm{dp} 0,64)$; adicionam à formação novos conhecimentos não adquiridos em sala de aula $(4,70 \mathrm{dp}$ $0,70)$; oportunizam conhecer a realidade local do sistema de saúde $(4,77 \mathrm{dp}$ 0,59 ); e contribuem para a formação de um profissional mais humano, ético e comprometido com a saúde pública $(4,74$ dp 0,58).

Afirmativas que abordaram aspectos relacionais também foram bem avaliadas pelos discentes. Por meio da percepção relatada, observou-se que os estágios propiciam o contato e a troca de experiências com profissionais de

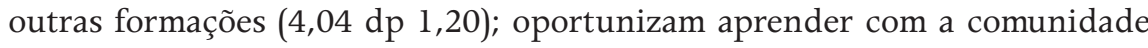
que usa o serviço $(4,40 \mathrm{dp}$ 0,97); contribuem para a aquisição de habilidades e competências de comunicação $(4,72 \mathrm{dp}$ 0,61); favorecem a reflexão e o questionamento das ações, a fim de, juntamente com a equipe de saúde, solucionarem impasses e conflitos $(4,31 \mathrm{dp} 0,94)$. Em nenhum dos itens houve diferença significativa entre as IESs.

Vivenciar os cenários de prática, com os quais o discente poderá se deparar no futuro exercício da profissão, mostra-se importante para a formação do perfil de um farmacêutico qualificado para o SUS. A análise revelou que os discentes de Farmácia das três IESs consentem que os estágios contribuem para sua formação profissional. Ademais, eles consideram o estágio como uma atividade que potencializa o vínculo do estudante com o SUS, despertando seu interesse em ingressar nos serviços públicos de saúde após concluir sua

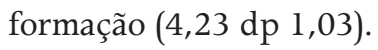


A percepção dos discentes sobre estágios e vivências nos serviços públicos de saúde costuma ser positiva também para os demais cursos da saúde. Estes ciclos de formação possibilitam aos discentes vivenciarem, na prática, o funcionamento do sistema. Eles permitem a interação ativa com a população e com os profissionais de saúde; a observação das fragilidades; e a visualização tanto dos processos de trabalho quanto das dificuldades enfrentadas pela equipe de saúde (Emmi, Silva e Barroso, 2017; Couto et al., 2018). Segundo Emmi, Silva e Barroso (2017), os estágios realizados em serviços do SUS oportunizam a aquisição de competências e habilidades para o trabalho neste sistema, bem como estimulam a capacidade crítica dos discentes para detectarem as mudanças necessárias no serviço.

A proposta de reorientação da formação em saúde, na lógica da promoção de vivências de estudantes em espaços do serviço, tem concretizado experiências promissoras de educação. Estas experiências destacam-se por desenvolver competências essenciais naqueles que irão atuar no SUS, como a resolução de problemas em equipe e a comunicação interprofissional (Figueredo et al., 2018), resultados que o presente estudo corrobora. Os estágios oportunizam contato e trocas de experiências, proporcionam relações interprofissionais e o trabalho em equipe multiprofissional.

Segundo Faria et al. (2018), as vivências dos estudantes em serviços têm dado condições para que eles, em suas relações com o outro, assumam experiências e realidades como seres sociais, transformadores e críticos. Em outras palavras, as vivências oportunizam aos estudantes exercitarem suas capacidades de reflexão e de aprenderem fazendo, tornando-se sujeitos da ação durante o processo de aprendizagem. Segundo os mesmos autores, para uma formação profissional em saúde condizente com as demandas e necessidades do SUS, é fundamental a inserção dos discentes e dos docentes na rede local de saúde.

Os marcos legais delegam ao SUS a ordenação e a formação de profissionais de saúde (Brasil, 1988; 1990). Cabe-lhe buscar a transformação e a qualificação da atenção à saúde, dos processos formativos e das práticas de educação em saúde (Brasil, 2004). Algumas possibilidades foram encontradas por este estudo, visando ao fortalecimento da formação farmacêutica e ao preparo dos discentes para o trabalho no SUS: aumentar a oferta de disciplinas com vivências nos serviços públicos de saúde; diversificar os cenários de prática durante os estágios, contemplando todos os níveis de complexidade do sistema de saúde; ampliar as vagas e a participação dos discentes em estágios obrigatórios e não obrigatórios em cenários da saúde pública; e fortalecer as relações interpessoais entre supervisores, preceptores e estudantes em estágio. 


\section{Conhecimentos e habilidades para o trabalho no SUS e fatores associados}

Afirmativas relativas aos conhecimentos e às habilidades para atuação no SUS tiveram variação, entre as IESs, quanto ao grau de concordância autorrelatada pelos discentes (Tabela 3). A IES3 apresentou médias significativamente inferiores às outras IESs nas questões que abordaram habilidades de comunicação para o trabalho multiprofissional $(3,40 \mathrm{dp} 1,00)$; preparo para atuação na atenção básica (3,04 dp 1,00); e preparo para atuação na média e alta complexidade $(2,76 \mathrm{dp} 0,96)$. A IES3 também evidenciou média significativamente inferior para a questão sobre os conhecimentos da estrutura da AF e das atividades do profissional farmacêutico no SUS $(3,66 \mathrm{dp} 0,90)$, quando comparada com a média da IESI $(4,10 \mathrm{dp}$ 0,63).

Tabela 3

Concordância (média [dp]) com as afirmativas relativas a conhecimentos e habilidades para a atuação no SUS, por instituição de ensino superior, faixa etária, período do curso e estágio. Região metropolitana de Porto Alegre, RS, Brasil, 2018

\begin{tabular}{|c|c|c|c|c|c|c|c|}
\hline & & $\begin{array}{l}\text { Conheço o } \\
\text { SUS, seus } \\
\text { princípios e } \\
\text { diretrizes, sua } \\
\text { estrutura e } \\
\text { sua rede de } \\
\text { serviços. }\end{array}$ & $\begin{array}{l}\text { Conheço } \\
\text { como é } \\
\text { estruturada } \\
\text { a AF no SUS } \\
\text { assim como } \\
\text { as atividades } \\
\text { do profissional } \\
\text { Farmacêutico. }\end{array}$ & $\begin{array}{l}\text { Estou } \\
\text { confiante } \\
\text { com minhas } \\
\text { habilidades } \\
\text { de } \\
\text { comunicação } \\
\text { para trabalhar } \\
\text { em ambiente } \\
\text { multiprofis- } \\
\text { sional. }\end{array}$ & $\begin{array}{l}\text { Estou pre- } \\
\text { parado para } \\
\text { atuar como } \\
\text { profissional } \\
\text { Farmacêutico } \\
\text { do SUS na AB. }\end{array}$ & $\begin{array}{l}\text { Estou pre- } \\
\text { parado para } \\
\text { atuar como } \\
\text { profissional } \\
\text { Farmacêutico } \\
\text { do SUS na } \\
\text { MAC. }\end{array}$ & $\begin{array}{l}\text { Quero tra- } \\
\text { balhar como } \\
\text { profissional } \\
\text { Farmacêutico } \\
\text { do SUS. }\end{array}$ \\
\hline \multirow[t]{4}{*}{ Universidade } & p-valor & 0,068 & 0,029 & 0,000 & 0,000 & 0,001 & 0,135 \\
\hline & IES1 & $4,12(0,76)^{\mathrm{a}}$ & $4,10(0,63)^{a}$ & $4,15(0,77)^{a}$ & $3,80(0,94)^{a}$ & $3,42(1,13)^{\mathrm{a}}$ & $3,42(1,45)^{a}$ \\
\hline & IES2 & $3,92(0,94)^{a}$ & $3,92(0,97)^{\mathrm{ab}}$ & $3,92(0,91)^{\mathrm{a}}$ & $3,65(0,97)^{a}$ & $3,42(1,08)^{\mathrm{a}}$ & $3,74(1,39)^{a}$ \\
\hline & IES3 & $3,75(0,76)^{\mathrm{a}}$ & $3,66(0,90)^{b}$ & $3,40(1,00)^{b}$ & $3,04(1,00)^{b}$ & $2,76(0,96)^{\mathrm{b}}$ & $3,16(1,46)^{a}$ \\
\hline \multirow[t]{4}{*}{ Faixa etária } & p-valor & 0,449 & 0,126 & 0,018 & 0,001 & $<0,001$ & 0,059 \\
\hline & $18-25$ & $3,87(0,79)^{a}$ & $3,73(0,83)^{\mathrm{a}}$ & $3,55(0,92)^{a}$ & $3,15(1,00)^{\mathrm{a}}$ & $2,82(0,99)^{a}$ & $3,14(1,43)^{a}$ \\
\hline & $26-35$ & $3,92(0,88)^{\mathrm{a}}$ & $4,04(0,84)^{\mathrm{a}}$ & $3,96(1,02)^{\mathrm{b}}$ & $3,80(0,94)^{b}$ & $3,49(1,08)^{b}$ & $3,69(1,46)^{a}$ \\
\hline & $36-45$ & $4,25(0,71)^{\mathrm{a}}$ & $3,88(1,36)^{a}$ & $4,25(1,04)^{b}$ & $3,88(1,13)^{b}$ & $4,00(1,07)^{c}$ & $3,88(1,36)^{a}$ \\
\hline \multirow[t]{2}{*}{$\begin{array}{l}\text { Período do } \\
\text { curso }\end{array}$} & p-valor & 0,933 & 0,052 & 0,944 & 0,131 & 0,025 & 0,000 \\
\hline & $\begin{array}{l}3^{\circ}-4^{\circ} \\
\text { ano }\end{array}$ & $3,90(0,80)^{a}$ & $3,71(0,92)^{\mathrm{a}}$ & $3,72(1,01)^{a}$ & $3,27(1,09)^{a}$ & $2,91(1,10)^{\mathrm{a}}$ & $2,99(1,51)^{a}$ \\
\hline
\end{tabular}



instituição de ensino superior, faixa etária, período do curso e estágio. Região metropolitana de Porto Alegre, RS, Brasil, 2018.

\begin{tabular}{|c|c|c|c|c|c|c|c|}
\hline & $\begin{array}{l}\text { Último } \\
\text { ano }\end{array}$ & $3,89(0,83)^{\mathrm{a}}$ & $3,98(0,80)^{\mathrm{a}}$ & $3,74(0,95)^{\mathrm{a}}$ & $3,53(0,96)^{\mathrm{a}}$ & $3,31(1,03)^{b}$ & $3,81(1,25)^{b}$ \\
\hline \multirow[t]{3}{*}{ Estágio } & p-valor & 0,716 & 0,078 & 0,005 & 0,031 & 0,022 & 0,000 \\
\hline & Sim & $3,91(0,83)^{\mathrm{a}}$ & $3,95(0,78)^{\mathrm{a}}$ & $3,91(0,88)^{a}$ & $3,54(1,00)^{\mathrm{a}}$ & $3,26(1,03)^{\mathrm{a}}$ & $3,75(1,34)^{a}$ \\
\hline & Não & $3,86(0,80)^{\mathrm{a}}$ & $3,68(0,97)^{\mathrm{a}}$ & $3,44(1,05)^{\mathrm{b}}$ & $3,17(1,05)^{\mathrm{b}}$ & $2,85(1,13)^{b}$ & $2,78(1,42)^{b}$ \\
\hline Total & & $3,90(0,82)$ & $3,84(0,87)$ & $3,73(0,98)$ & $3,39(1,03)$ & $3,10(1,08)$ & $3,37(1,45)$ \\
\hline
\end{tabular}

Fonte: Os autores.

Nota: Médias seguidas de letras iguais na coluna não diferem entre si $(p<0,05)$. Teste de Tukey. IES: instituição de ensino superior, AF: Assistência Farmacêutica, AB: Atenção Básica, MAC: Média e Alta Complexidade, SUS: Sistema Único de Saúde.

Estudo semelhante, realizado em todas as escolas de medicina do Reino Unido, com médicos formados em 2011 e 2012 e questionados um ano após concluírem sua formação acadêmica, mostrou que a percepção autorrelatada do preparo para o trabalho tem aumentado em quase todas as escolas de medicina do Reino Unido (Lachish, Goldacre e Lambert, 2016). No mesmo estudo, Lachish, Goldacre e Lambert (2016) identificaram diferenças na percepção dos egressos em relação às escolas de medicina. Quase todos os egressos das escolas mais bem avaliadas concordaram estarem bem preparados para o trabalho clínico, porém apenas metade dos egressos das escolas não tão bem avaliadas externaram igual percepção.

Devido às diferenças verificadas entre instituições de ensino, os autores do referido estudo concluíram que muitos fatores, além dos currículos e das políticas de educação, podem contribuir para o ambiente de aprendizado (Lachish, Goldacre e Lambert, 2016). Na presente investigação, as variáveis vínculo empregatício, atividades de vivência nos serviços de saúde pública, e período em que se encontram os alunos no curso mostraram diferenças significativas na comparação entre IESs. Além da singularidade dos cursos, tais fatores possivelmente contribuíram para as diferenças encontradas entre as IESs, nas percepções autorrelatadas de preparo para o trabalho no SUS.

As análises das variáveis: faixa etária, período do curso e realização de estágios no SUS demonstraram significativo poder de alteração no grau de concordância autorrelatado para as questões relativas aos conhecimentos e às habilidades para o trabalho no SUS. Discentes das faixas etárias 26-35 e 36-45 anos relataram sentir maior confiança com as habilidades de comunicação e estarem mais bem preparados para atuarem na $\mathrm{AB}$, quando comparados com os da faixa etária de 18-25 anos. O aumento da faixa etária coincide com o aumento, na percepção autorrelatada, de preparo para a atuação na MAC (Tabela 3). 
Quanto às demais variáveis, discentes do último ano do curso sentem-se mais preparados para atuarem na $\operatorname{MAC}(3,31 \mathrm{dp} 1,03)$ e expressam maior desejo em trabalhar no SUS $(3,81 \mathrm{dp} 1,25)$, em comparação com os do terceiro e quarto anos. Discentes que realizaram estágio(s) no SUS sentem-se mais confiantes em suas habilidades de comunicação para trabalhar em ambiente multiprofissional $(3,91 \mathrm{dp} 0,88)$, mais preparados para atuar na $\mathrm{AB}(3,54 \mathrm{dp}$

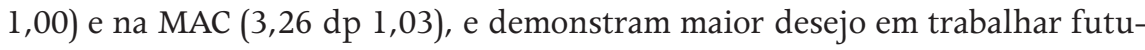
ramente no SUS $(3,75 \mathrm{dp} 1,34)$. As ações ou atividades de extensão universitária, isoladamente, não alteraram significativamente o grau de concordância para estas questões.

Ao corroborar os resultados encontrados, Sumpradit et al. (2014) observaram diferenças nas competências profissionais autorrelatadas por egressos dos cursos de Farmácia na Tailândia. Egressos do programa Doctor of Pharmacy, com extensão de seis anos e duas mil horas de experiência nos serviços, relataram maiores competências em serviços de cuidado intensivo, serviços de reconciliação medicamentosa e serviços de atenção primária. Egressos do programa Bachelor of Science in Pharmacy, com cinco anos de extensão e apenas quinhentas horas de experiência em serviços, relataram menores competências para estas mesmas atividades. Por conseguinte, o contato direto com os serviços, durante o período de formação acadêmica, mostra-se relevante para a ampliação da autopercepção sobre o preparo para o trabalho.

A força de associação das variáveis na alteração do conjunto das questões relativas aos conhecimentos e às habilidades para atuação no SUS, foi igualmente analisada. Na análise bivariada, encontrou-se associação positiva e estatisticamente significativa para autopercepção de preparo para o trabalho no SUS e as seguintes variáveis: IES, faixa etária, estado civil, estágio obrigatório no SUS, estágio não obrigatório no SUS, estágio na MAC e vínculo empregatício. Contudo, após a realização da análise multivariada, apenas o fator idade manteve associação positiva e estatisticamente significativa, em que discentes de 36 a 45 anos sentiram-se 2,53 vezes mais preparados para atuarem no SUS na comparação com aqueles de até 25 anos (Tabela 4). 
Tabela 4

Somatório do grau de concordância autorrelatado para as afirmativas relativas a conhecimentos e habilidades para a atuação no SUS. Distribuição das razões de prevalência (RP), bivariada e multivariada (regressão de Poisson), e seus respectivos intervalos de 95\% de confiança (IC95\%), segundo as variáveis do estudo. Região metropolitana de Porto Alegre, RS, Brasil, 2018.

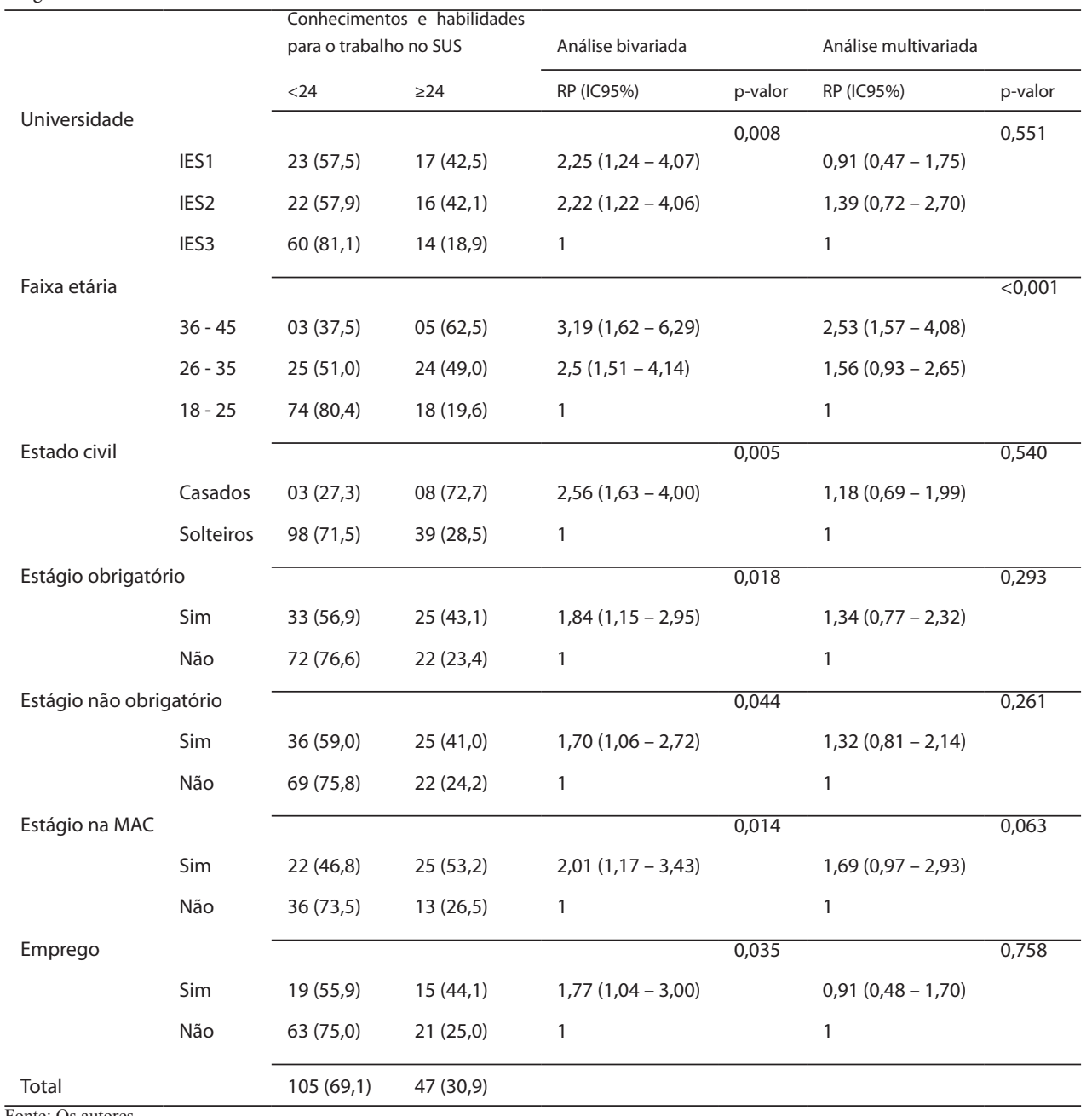

Fonte: Os autores.

IES: instituição de ensino superior, AF: Assistência Farmacêutica, AB: Atenção Básica, MAC: Média e Alta Complexidade, SUS: Sistema Único de Saúde. 
Apesar de a variável idade, dentre as variáveis analisadas, mostrar força para alterar significativamente o somatório das questões de autopercepção sobre o preparo para o trabalho no SUS, este resultado não expressa consenso. Resultados divergentes em relação ao presente estudo foram encontrados por Woods et al. (2015), ao analisarem a autoconfiança de estudantes de enfermagem sobre o preparo para a atuação em um contexto com múltiplos pacientes. Os autores identificaram que a confiança estava inversamente associada à idade. Da mesma forma, Rocha, Leles e Queiroz (2018) encontraram resultados divergentes em relação ao presente estudo, ao analisarem o desempenho de estudantes de nutrição, no Exame Nacional de Desempenho dos Estudantes (Enade). A variável idade demonstrou correlação negativa significativa para o desempenho acadêmico.

Questões provenientes das experiências adquiridas durante a vida, muitas delas análogas às de trabalho, podem explicar as melhores autopercepções dos discentes mais velhos relativas a seu preparo para o trabalho no SUS. Henrique et al. (2018), ao analisarem a autopercepção de professores de educação física sobre suas competências para o trabalho, mostraram haver diferenças significativas nas percepções de professores iniciantes versus professores experientes. Segundo tais autores, profissionais experientes, com idade maior e mais anos de experiência profissional, autopercebem-se mais competentes para o trabalho.

Todavia, a melhor autopercepção de preparo para o trabalho não implica necessariamente melhor desempenho profissional. Um estudo que investigou o impacto de uma intervenção educativa na confiança e na competência, durante a realização de uma tarefa cirúrgica simples por estudantes de medicina, descobriu que a confiança autorrelatada antes da intervenção estava inversamente relacionada ao desempenho objetivo, ou seja, maior confiança foi assocada a pior desempenho (Leopold et al., 2005). São necessários estudos similares a este referido para que haja melhor entendimento da autopercepção dos discentes sobre o preparo para o trabalho em saúde, por permitirem avaliar se sua percepção de confiança está alinhada a suas reais habilidades e capacidades no contexto da prática profissional.

As variáveis gênero, ensino público ou privado, período em que se encontra o discente no curso, atividades de extensão universitária ou pesquisa e supervisão de estágio não demonstraram ter impacto no conjunto de questões sobre as percepções autorrelatadas pelos discentes quanto ao preparo para atuação nos serviços públicos de saúde. 


\section{Considerações finais}

Este estudo analisou, em três IESs localizadas na RMPA, o currículo do curso de Farmácia e a percepção dos respectivos acadêmicos no tocante a ações de integração ensino e serviços públicos de saúde desenvolvidas durante o processo de formação acadêmica.

As percepções identificadas, relatadas pelos discentes, contribuíram para ampliar a compreensão acerca da formação generalista de farmacêuticos, das formas efetivas de contato com os serviços públicos de saúde, dos fatores que interferem de maneira positiva na formação de um perfil profissional para o SUS. O estudo também contribuiu para o preparo de subsídios, visando ao processo de elaboração ou reformulação curricular.

Os resultados encontrados sinalizam as potencialidades dos estágios, em cenários de saúde pública, para a formação de profissionais farmacêuticos com perfil para o trabalho no SUS. Os estágios em saúde pública, na visão dos discentes, contribuem para a formação profissional, provendo-os de habilidades, atitudes e conhecimentos específicos para a atuação em saúde pública.

Observou-se que a idade é um fator relevante para o reconhecimento das competências e habilidades necessárias para o trabalho. Pressupõe-se que discentes com mais idade autorrelatam melhores competências para o trabalho, devido às diversificadas experiências por eles vivenciadas. Contudo, são necessários outros estudos para esclarecimento das contradições encontradas nas comparações entre idade, desempenho e percepção de preparo para o trabalho, inclusive de perfis psicológicos e geracionais.

Os resultados do presente estudo sugerem que a formação de um perfil profissional adequado ao SUS não depende unicamente das instituições formais de ensino ou das disciplinas teórico práticas por elas ofertadas. As relações de trabalho em ambiente multiprofissional, os níveis de complexidade do sistema de saúde, as interações dos profissionais com a comunidade exigem uma ampla formação ancorada na realidade local de saúde.

Mimetizar as experiências a que esses estudantes vão se expor durante sua vida, principalmente as relacionais, no decorrer da formação acadêmica em Farmácia, surge como uma maneira inovadora de formar profissionais para o SUS. Vivências nos cenários de prática, em semestres iniciais do curso, proporcionando a integração precoce dos discentes com os serviços de saúde, podem catalisar os efeitos positivos da idade no reconhecimento das competências necessárias para o trabalho no SUS. 


\section{Colaboradores}

Lucas Balsanelli Souza foi responsável pela concepção da ideia original, redação, discussão dos resultados, revisão e aprovação da versão final. Andréa Wander Bonamigo orientou a pesquisa, colaborou na concepção da ideia original, na revisão e na aprovação da versão final. Não há conflito de interesses.

\section{INTEGRACIÓN ENSEÑANZA-SERVICIO EN LA FORMACIÓN DE PROFESIONALES PARA SISTEMAS PÚBLICOS DE SALUD}

Resumen La integración enseñanza-servicio, entendida por el trabajo articulado de estudiantes, profesores y trabajadores en escenario de práctica, tiene por finalidad la excelencia de la formación profesional y la calidad del trabajo en salud. El objetivo de este estudio es analizar, por medio de la percepción de los discentes, la integración enseñanza-servicio, que ocurre durante el período de graduación en Farmacia. Se trata de un estudio cuantitativo, conducido en 2018, caracterizado como relevamiento de corte transversal, en el cual se aplicó un cuestionario estructurado. Participaron del estudio 155 académicos de cursos de Farmacia, matriculados en tres universidades, dos públicas y una privada, localizadas en la región metropolitana de Porto Alegre, Rio Grande do Sul, Brasil. Los resultados encontrados mostraron las potencialidades de las prácticas en escenarios de salud pública para la formación de profesionales farmacéuticos más confiados para el futuro trabajo en el Sistema Único de Salud. Los datos obtenidos revelaron la variable edad de los discentes como factor relevante para el reconocimiento de las competencias necesarias para el trabajo. Tales hallazgos pueden contribuir para el proceso de elaboración o reformulación curricular de los cursos de Farmacia, con vistas a la formación de un profesional crítico, reflexivo y conocedor de la realidad de salud local.

Palabras clave educación en salud; educación en farmacia; servicios de integración docenteasistencial; Sistema Único de Salud.

\section{Nota}

1 Artigo baseado na dissertação de mestrado de Lucas Balsanelli Souza intitulada "Formação Farmacêutica: integração ensino-serviço dos acadêmicos de Farmácia com o Sistema Único de Saúde", apresentada ao Programa de Pós-Graduação em Ensino na Saúde, da Universidade Federal de Ciências da Saúde de Porto Alegre, em 2019. 


\section{Referências}

BRASIL. Constituição da República Federativa do Brasil de 1988. Diário Oficial [da] República Federativa do Brasil, Poder Executivo, Brasília, DF, 1988.

BRASIL. Lei $\mathrm{n}^{\circ} 8.080$, de 19 de setembro de 1990. Dispõe sobre as condições para a promoção, proteção e recuperação da saúde, a organização e o funcionamento dos serviços correspondentes e dá outras providências. Diário Oficial [da] República Federativa do Brasil, Poder Executivo, Brasília, DF, 1990.

BRASIL. Lei $\mathrm{n}^{\circ}$ 9.394, de 20 de dezembro de 1996. Estabelece as diretrizes e bases da educação nacional. Diário Oficial [da] República Federativa do Brasil, Poder Executivo, Brasília, DF, 1996.

BRASIL. Ministério da Educação. Conselho Nacional de Educação. Câmara de Educação Superior. Resolução CNE/CES n. 2, de 19 de fevereiro de 2002. Institui diretrizes curriculares nacionais do curso de graduação em Farmácia. Diário Oficial [da] República Federativa do Brasil, Poder Executivo, Brasília, DF, 2002.

BRASIL. Portaria $n^{\circ}$ 198/GM em 13 de fevereiro de 2004. Institui a Politica Nacional de Educação Permanente em Saúde como estratégia do Sistema Único de Saúde para a formação e o desenvolvimento de trabalhadores para o setor e dá outras providências. Brasília, 2004.

BRASIL. Ministério da Educação. Conselho Nacional de Educação. Câmara de Educação Superior. Resolução $n^{\circ}$ 6, de 19 de outubro de 2017. Institui as Diretrizes Curriculares Nacionais do Curso de Graduação em Farmácia e dá outras providências. Diário Oficial [da] República Federativa do Brasil, Poder Executivo, Brasília, DF, 2017, p 30.

BRASIL. Ministério da Educação. Cadastro Nacional de Cursos e Instituições de Educação Superior Cadastro e-MEC. 2018a. Disponível em: <http://emec.mec.gov.br/>. Acesso em: 20 dez. 2018a.
BRASIL. Edital nº 10, 23 de julho 2018 seleção para o programa de educação pelo trabalho para a saúde pet-saúde/interprofissionalidade: 2018/2019. Diário Oficial [da] República Federativa do Brasil, Poder Executivo, Brasília, DF, 24 jul. 2018b. Seção 3, p. 78.

CAVALHEIRO, Maria T. P.; GUIMARÃES, Alóide L. Formação para o SUS e os desafios da integração ensino serviço. Caderno FNEPAS, São Paulo, v. 1, p. 19-27, 2011.

CECCIM, Ricardo B.; BRAVIN, Fábio P.; SANTOS, Alexandre A. D. Educação na saúde, saúde coletiva e ciências políticas: uma análise da formação e desenvolvimento para o Sistema Único de Saúde como política pública. Lugar Comum, [s.1.], n. 28, p. 159-180, 2009.

COUTO, Vanessa B. M. et al. Vivenciando a Rede: Caminhos para a Formação do Médico no Contexto do SUS. Revista Brasileira de Educação Médica, Brasília, v. 42, n. 2, p. 5-14, 2018. Disponível em: < http://dx.doi. org/10.1590/1981-52712015v42n2rb2016107 >. Acesso em: 20 nov. 2018.

CRESWELL, John W. Projeto de pesquisa: métodos qualitativo, quantitativo e misto. 3. ed. Porto Alegre: Artmed, 2010.

DAMIANCE, Patrícia R. M. et al. Formação para o SUS: uma análise sobre as concepções e práticas pedagógicas em saúde coletiva. Trabalho, Educação e Saúde, Rio de Janeiro, v. 14, n. 3, p. 699-721, 2016. Disponível em: <http://dx.doi. org/10.1590/1981-7746-sol00014>. Acesso em: 5 nov. 2018.

EMMI, Danielle T.; SILVA, Daiane M. C. D.; BARROSO, Regina F. F. Experiência do ensino integrado ao serviço para formação em Saúde: percepção de alunos e egressos de Odontologia. Interface: Comunicação, Saúde, Educação, Botucatu, v. 22, n. 64, p. 223-236, 2017. Disponível em: <http://dx.doi. org/10.1590/1807-57622016.0655>. Acesso em: 2 nov. 2018. 
FARIA, Lina et al. Integração ensino-serviçocomunidade nos cenários de práticas na formação interdisciplinar em Saúde: uma experiência do Programa de Educação pelo Trabalho para a Saúde (PET-Saúde) no sul da Bahia, Brasil. Interface: Comunicação, Saúde, Educação, Botucatu, v. 22, n. 67, p. 1.2571.266, 2018. Disponível em: <http://dx.doi. org/10.1590/1807-57622017.0226>. Acesso em: 15 dez. 2018.

FIGUEREDO, Wilton N. et al. Práticas colaborativas nas urgências em Saúde: a interprofissionalidade do Programa PermanecerSUS, Secretaria Estadual de Saúde da Bahia, Brasil ${ }^{*}$. Interface: Comunicação, Saúde, Educação, Botucatu, v. 22, sup. 2, p. 1.697-1.704, 2018. Disponível em: $<$ http://dx.doi.org/10.1590/180757622017.0678>. Acesso em: 8 dez. 2018.

HENRIQUE, José et al. Autopercepção de competências profissionais de professores de educação física iniciantes e experientes. Revista Brasileira de Ciências do Esporte, Brasília, v. 40, n. 4, p. 388-396, 2018. Disponível em: <http://www.sciencedirect.com/science/ article/pii/S0101328916301433>. Acesso em: 8 dez. 2018.

LACHISH, Shelly; GOLDACRE, Michael J.; LAMBERT, Trevor. Self-reported preparedness for clinical work has increased among recent cohorts of UK-trained first-year doctors. Postgraduate Medical Journal, Londres, v. 92, n. 1.090, p. 460, 2016. Disponível em: < http://pmj.bmj.com/content/92/1090/460. abstract $>$. Acesso em: 10 out. 2018.

LEOPOLD, Seth S. et al. Impact of educational intervention on confidence and competence in the performance of a simple surgical task. Journal of Bone and Joint Surgery, Needham, v. 87, n. 5, p. 1.031-1.037, maio 2005.

MACHADO, Maria H.; XIMENES NETO, Francisco R. G. Gestão da Educação e do Trabalho em Saúde no SUS: trinta anos de avanços e desafios. Ciência e Saúde Coletiva, Rio de
Janeiro, v. 23, n. 6, p. 1.971-1.979, 2018. Disponível em: <http://dx.doi.org/10.1590/1413$81232018236.06682018>$. Acesso em: 10 out. 2018.

PEDUZZI, Marina et al. Educação interprofissional: formação de profissionais de saúde para o trabalho em equipe com foco nos usuários. Revista da Escola de Enfermagem da USP, São Paulo, v. 47, n. 4, p. 977-983, 2013. Disponível em: <http:// dx.doi.org/10.1590/S0080-623420130000400029> Acesso em: 9 out. 2018.

ROCHA, Aline L. D. P.; LELES, Claudio R.; QUEIROZ, Maria G. Fatores associados ao desempenho acadêmico de estudantes de Nutrição no Enade. Revista Brasileira de Estudos Pedagógicos, Brasília, v. 99, n. 251, p. 74-94, 2018. Disponível em: <http://dx.doi. org/10.24109/2176-6681.rbep.99i251.3162>. Acesso em: 23 nov. 2018.

SOUZA, Lucas B.; BONAMIGO, Andrea W. A interface ensino-serviço na formação farmacêutica: revisão integrativa. Saúde em Redes, Porto Alegre, v. 4, n. 2, 2018.

SPADA, Celso et al. Farmácia. In: HADDAD, Ana E., et al (Ed.). A trajetória dos cursos de graduação na área da saúde: 1991-2004. Brasília: Instituto Nacional de Estudos e Pesquisas Educacionais Anísio Teixeira, 2006. p. 531.

SUMPRADIT, Nithima et al. Comparison of self-reported professional competency across pharmacy education programs: a survey of Thai pharmacy graduates enrolled in the public service program. Advances in medical education and practice, Auckland, v. 5, p. 347-357, 2014. Disponível em: <https:// www.ncbi.nlm.nih.gov/pubmed/25337000>. Acesso em: 15 out. 2018

WOODS, Cindy et al. Undergraduate student nurses' self-reported preparedness for practice. Collegian, Chatswood, v. 22, n. 4, p. 359-368, 2015. Disponível em: <http:// dx.doi.org/10.1016/j.colegn.2014.05.003>. Acesso em: 14 out. 2018. 